\title{
Monitoring and Management of Natural Territorial Complexes
}

\author{
Dr. Ghazi H. Shakah ${ }^{1, *}$, Victor Kras nopros hin ${ }^{2}$, Aleksandr Valvachev ${ }^{3}$ \\ ${ }^{1}$ Dean of the Faculty of Computer and Information Technology, Ajloun National University, Jordan \\ ${ }^{2}$ Dept. Information Management Sy stems, Belarusian State University, Minsk, Belarus \\ ${ }^{3}$ Dept. of Information Management Systems, Belarusian State University, Minsk, Belarus
}

\begin{abstract}
The paper describes the use of organization and graph theory for constructing model that management distributed nature and artificial beings. It's proposed a virtual model of the organization, focused on the implementation of monitoring, regard less of the size and remoteness of the objects of observation from the center. The algorithm of its construction, based on the representation of the model in the form of attributed tree, is described. The resulting virtual model can be considered as a basis for solving a nu mber of applications for monitoring, including decision-making, knowledge acquisition, visualization of large structures, the construction of dynamic digital maps, integration with other systems, etc.
\end{abstract}

Keywords Environmental Monitoring, Distributed Organizations, Attributive Tree

\section{Introduction}

Rapid changes in the environment, caused by climate change and globalization, have led to a nu mber of large-scale economic, social and environmental problems. One of them is a rapid change in ecology of natural-territorial co mplexes. As a result, there are difficulties in agriculture, disappearance of many species of animals and plants, deterioration of health and quality of life in whole[1,2].

Under natural-territorial complexes (NTC) can be understood any selected area in some respects, including natural and artificial objects that are observed and evaluated. For a quick and adequate response to changes in NTC have to learn how to fix the changes quickly, to obtain relevant information, to assess the situation quickly and take appropriate decisions. Unfortunately, technology intended for this are just being created, so it is still impossible to estimate the real man-made disasters, including the largest in the history disaster in the Chernobyl NPP. A similar situation is emerging after the accident at the NPP in Japan.

Traditionally, monitoring of NTC is performed in the framework of environmental monitoring. Environmental monitoring is a comprehensive system of observation of the state of environment, assessment and forecast of environmental changes caused by natural and anthropogenic factors. Depending on the scale of observation they distinguish local, regional and global mon itoring[3].

* Corresponding author:

warsaw2000@yahoo.com (Dr. Ghazi H. Shakah)

Published online at http://journal.sapub.org/ac

Copyright (C) 2012 Scientific \& Academic Publishing. All Rights Reserved
Organizational structure, implementing mon itoring (MSO), includes: a regional station for the initial monitoring, the base station, which aggregates information from regions to the national center. These national centers are part of inter-state environmental organizations.

The most important tasks of monitoring are:

1) a rational distribution of network of mon itoring stations across the globe;

2) provision with a system of devices and methods of operational control of environ mental quality;

3) creation of a clear hierarchical system of data collection, storage, transmission, processing and generalization of information on regional, national and global levels $[1,3]$.

Creating of MSO for solving these problems is complex, multistage and expensive problem, exacerbated by an increase in the scale of the observed NTC.

Changing of boundaries and climatic changes have led to the need to reorganize the existing NTC and to create new NTC, comprising several countries (such as the nature reserve Bialowezhskaja Pushia, which is located on the territory of Belarus and Poland.). As a result, a problem of building of more efficient and less expensive MSO has become urgent $[2,4]$.

This rather complex organizational and technological problem includes a number of scientific and technical problems. One of them is to build a model for rapid building of MSO and information exchange among its participants regardless of their geographic location. This paper describes a possible variant of its decision on the basis of a synthesis of elements of organization theory and graph theory.

\section{Statement of the Problem}


This problem statement initially is technological in nature. Let there be a NTC (W). Monitoring of NTC should be implemented by the organization (S), which includes a set of geographically distributed regional stations. State of NTC is characterized by a final set of diagnostic indicators $(\mathrm{X})$.

It's required to develop a model of organizational structure (S) for monitoring NTC (W), which provides:

- formation of the participants of the monitoring;

- local identification of the staff in the admin istrative hierarchy;

- global identification of the staff in the Internet infrastructure.

- access of the center to cognitive resources;

- grouping and mapping of the structure of OSM in the graphics applications of various purposes, including geographical maps.

The main requirement for the solution: possibility to implement on standard personal IBM computers running with the OS Windows.

\section{Main Definitions}

To solve this problem is proposed:

- to consider the organization as a virtual structure composed of distributed objects, presented as a set of attributes;

- to introduce a concept of mobile mediator, providing information exchange between participants in a virtual organization in the global communications infrastructure.

The concept of virtuality means that the structure will be constructed in computer memory on base of the actual hierarchical structure (S), and the attributes of the structure will describe the actual characteris tics of (S), including indus trial, social, official and etc.

Formally, we represent the model of the virtual structure as a tuple:

$$
\mathrm{P}=\left(\mathrm{A}_{\mathrm{j}}^{\mathrm{i}} \text {, task, } \mathrm{m}, \mathrm{com}\right)
$$

where: $A_{j}{ }_{j}$ is attributes of an object, which makes part of the MSO; i, j are nu mbers of the object and attribute; (task) is the problem under consideration; $\mathrm{m}-$ is a mediator; co $\mathrm{m}-$ is communication[6].

Model (1) is universal in a determinate sense, as selecting different sets of attributes $\mathrm{A}_{\mathrm{j}}^{\mathrm{i}}$, and assigning appropriate values to them, you can simulate an organization of various configuration and composition.

For practical use of model (1) it's necessary to perform typing of attributes typical for monitoring. According to the statement of the problem, as these attributes can be considered the following attributes $[7,8]$ :

1) participant's status in the hierarchy (status). As a rule, the status defines the center (center), in itiating the task, experts $(\mathrm{E})$ and regional observers $(\mathrm{P})$, participating in its decision;

2) participant's identifier (name) (id);

3) the index of local (in the admin is trative hierarchy) positioning $\left(\mathrm{A}^{\mathrm{L}}\right)$;

4) the index of the global (Internet) positioning $\left(A^{G}\right)$;
5) the index for display of the structure of the virtual model in digital maps $\left(\mathrm{A}^{\mathrm{M}}\right)$. As a rule, this GPS - is coordinates of the object.

Let's adequately specify the model (1):

$$
\mathrm{P}=\left(\text { status,id, } \mathrm{A}^{\mathrm{L}}, \mathrm{A}^{\mathrm{G}}, \mathrm{A}^{\mathrm{M}} \text {, task, } \mathrm{m}, \text { com }\right)
$$

For practical use (1) we perform a detail of the component (task):

$$
\text { Task }=(\text { text, } \mathrm{X}, \mathrm{V}, \mathrm{U}, \mathrm{app})
$$

where: text is formulation of the problem; $\mathrm{X}$ is diagnostic variables; $\mathrm{V}$ is possible states of an object; $\mathrm{U}$ is a control for the object; app is equipment for measuring characteristics.

In accordance with (1) and (2) we construct a model of the med iator:

$$
\mathrm{m}=\left(\mathrm{A}^{\text {Gcenter }}, \mathrm{A}^{\mathrm{GP}}, \mathrm{Q}, \mathrm{X}\right)
$$

where: $\mathrm{Q}$ is inquiry to object $\mathrm{P}$ on the measurement of characteristics $\mathrm{X}$.

There is no need in adjustment of the communication component (com), as in most cases means of a standard e-mail are enough for movement of the mediator $\mathrm{m}$.

The proposed variant of the models (1) - (5), components of monitoring, allows going to development of a mechanism for constructing a virtual model of the organization.

\section{Model of Virtual MSO}

For practical use of models (1) - (4) it's necessary to choose a way of their presentation in a more "technological" form. For this propose it's proposed to use graph theory as it is a universal device for the presentation of hierarch ies of any $\operatorname{kind}[4,5]$.

Graph is a collection of objects (V) and ties between them (E):

$$
\mathrm{G}:=(\mathrm{V}, \mathrm{E})
$$

Accordingly, the objects $\mathrm{V}$ are called vertices or nodes of a graph, and ties E are arcs, or edges. A graph, any pair of vertices of which can be joined by routs, is called connected. A connected graph with no cycles (loops) is called a tree. A tree is called labeled (attributed) if its vertices or arcs are assigned with certain tags.

According to (2), in order to construct a model of virtual MSO, consisting of a center and a set of units (sub) in the form of a graph it is sufficient to perform the following operations:

- to create an admin is trative structure that provides scientific and technical solution of the complex tasks, including organization and support of the life-cycle monitoring NTC regardless of its size (Figure 1);

- to display the structure of MSO in the graph. It's enough to each unit MSO as sociate a vertex of the graph. The edges of the graph will match the flow of information between departments. Thus, a tree is formed; it's a model of organization structure (Figure 2). To construct the tree it is convenient to use standard tools of the program Visio.

- to mark the top of the tree. As a label we choose attribute $\mathrm{A}^{\mathrm{L}}$, as it reflects the hierarchy in a real organization, and mark the edges by the identifier of copies of mediator $\mathrm{m}$, 
providing communication between nodes (Figure 3). With a large number of units in the MSO, this operation takes a long time;



Figure 1. MSO structure

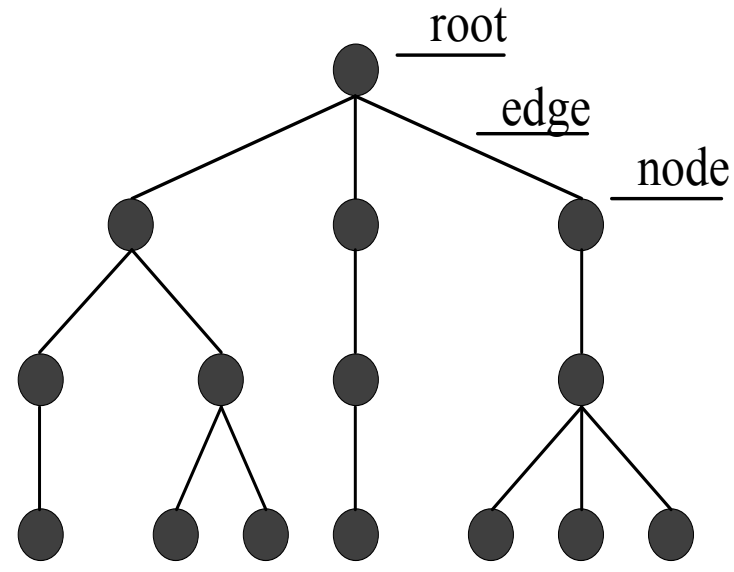

Figure 2. MSO representation

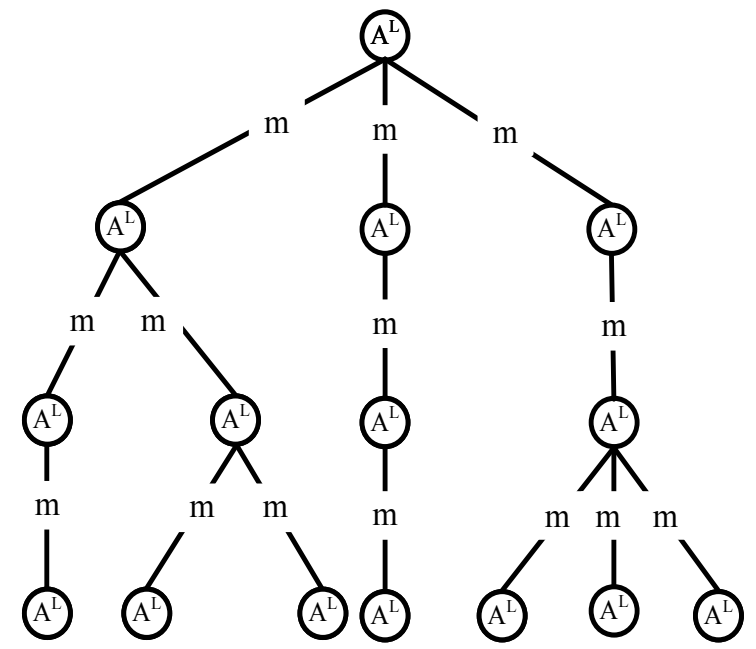

Figure 3. Tree skeleton

- to each vertex number we assign a set of attributes. In our case (according to model 2) these are global $\left(\mathrm{L}^{\mathrm{G}}\right)$, mapping $\left(\mathrm{A}^{\mathrm{M}}\right)$ and service $\left(\mathrm{A}^{\mathrm{F}}\right)$ attributes (Figure 4$)$. With a large number of units in the MSO, this operation is very laborious

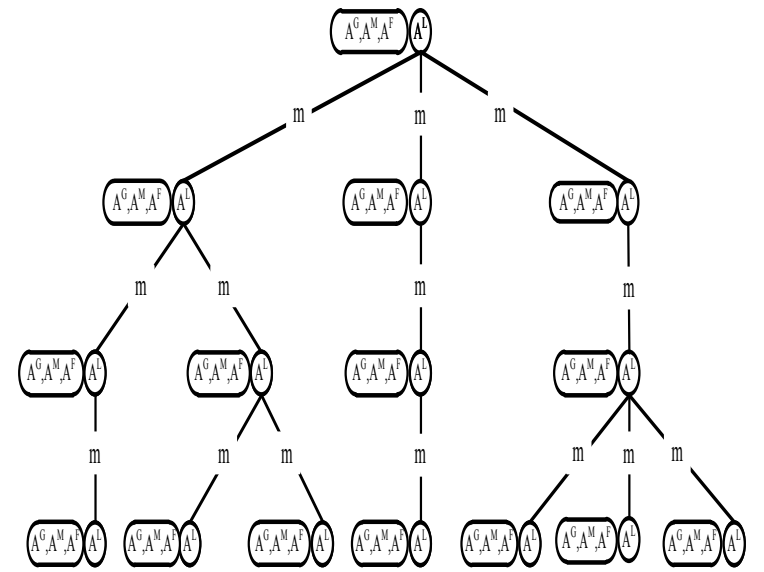

Figure 4. Attributed tree

Thus, the construction of the model MSO in the form of attributed tree of a virtual organization adds up to four steps:

- construction of a hierarchical scheme of a real organization;

- the mapping of this scheme in a tree;

- primary $\mathrm{A}^{\mathrm{L}}$-attribution of vertices and edges of the tree;

- secondary $\mathrm{A}^{\mathrm{G}}-, \mathrm{A}^{\mathrm{M}}-, \mathrm{A}^{\mathrm{F}}$ - attribution of vertices.

In practice, it is quite difficult to implement these steps, because the number of objects of the organization can reach tens or more, the nu mber of service attributes varies by tens, and the time for building of the branch of the virtual model for solving of the current operational task can be measured in hours.

These are only the most significant of the problems that impede the construction of monitoring systems for large-scale NTC.

To solve above mentioned problems, it's proposed a mechanis $m$ that automates the implementation of most labor-intensive operations.

\section{Mechanism of MSO Building}

A fast algorithm for constructing the frame of the model is developed. The algorithm is based on the abbreviated notation of the graph and the use of attributes from the previously created database.

The use of the notation and databases ensures automatic generation of the tree and a quick attribution of nodes.

Manual steps:

- to investigate the actual structure of MSO;

- to build a relevant sketch of graph model of virtual model;

- to perform the packaging on base of the sketch (for example, by Prufer or Gupta methods) and to get a shorten notation of graph model and formalize it as a string[5].

Automated stages:

- to read the notation;

- to unzip the notation (perform parsing of strings) and construct in memory an empty structure of the tree;

- to perform primary $\mathrm{A}^{\mathrm{L}}$-attribution;

- to consider the attributes $A^{\mathrm{G}}-, \mathrm{A}^{\mathrm{M}}-, \mathrm{A}^{\mathrm{F}}$-attribution of the 
database;

- to perform a secondary $\mathrm{A}^{\mathrm{G}}-, \mathrm{A}^{\mathrm{M}}-, \mathrm{A}^{\mathrm{F}}$-attribution;

- to record attributed tree in the database.

These algorithms differ from similar algorithms for packing of Prufer and Gupta by simplicity and possibility of automation in the design of sketches in Visio (as VSD-file).

The implementation of this algorithm does not exclude the manual labor, but significantly reduces its volume, because all labor-intensive processes of constructing of the skeleton of the tree and the attribution of vertexes are auto mated.

The algorithm allows displaying any hierarchical structure in virtual organization. Use of the notation and databases allows to shape the tree automatically and to make a quick attribution of nodes. $\mathrm{A}^{\mathrm{L}}-\mathrm{A}^{\mathrm{G}}$-, $\mathrm{A}^{\mathrm{M}}$-attributes are used respectively for the $\mathrm{v}$ isual as sess ment of the leadership of large organizational structures, global positioning of the structure and the construction of dynamic digital maps for different purposes, and $\mathrm{A}^{\mathrm{F}}$-attribute reflects traditional identifiers (address, name, e-signature and other features of the participants of the organization).

Virtual model of the organization is actually an environment for solution of a wide range of monitoring tasks, provides an access to remote participants monitoring, information exchange between them and the center.

In general, the solution generates an external "information-technological" view on the monitor in addition to the traditional "ecological" v iew.

\section{Information Exchange Mechanism}

A mobile structure is proposed for the organization of information exchange between the center and objects (sub)[9]. The structure includes parametric dialogue, intelligent mediator and delivery system (Figure 5). The intelligence of the mediator is the ability to ask questions, receive, analyze and store the answers.

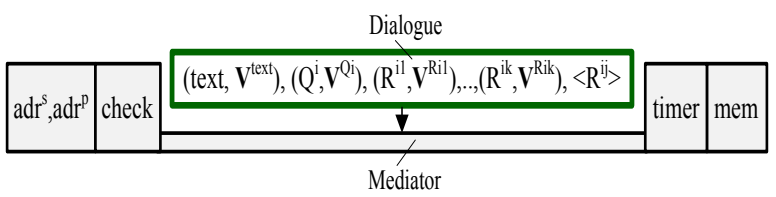

Figure 5. Mediator structure

The structure of the med iator includes: problem statement (text), question (Q), question variants (R) and the selected answer $(<\mathrm{R}>)$, as well as an optional visual support $(\mathbf{V})$. The structure of the delivery system comprises: the sender and recipient addresses $\left(\operatorname{adr}^{\mathrm{s}}, \operatorname{adr}^{\mathrm{p}}\right)$, means for controlling access to experts (check), timer (timer) to fix the time spent on the dialogue, and memory for storing results of the dialogue (mem). The structure is formed by the center and delivered to objects by means of the Internet. An array of information is formed during the dialogue between the mediator and the manager of the object. The information is encrypted and returned to the center.

An algorithm, which implements the process of knowledge acquisition, is developed. The algorith $\mathrm{m}$ builds a technologically oriented solution, including the identification of sources and dialogue setting to the type of the source. Automatic replacement of addresses and mailing of med iator copies ensured the possibility to parallelize the dialogue between the center and objects[7,8,9]. The general scheme of automation of monitoring on the basis of the offered models and algorithms is presented in Figure 6[9].

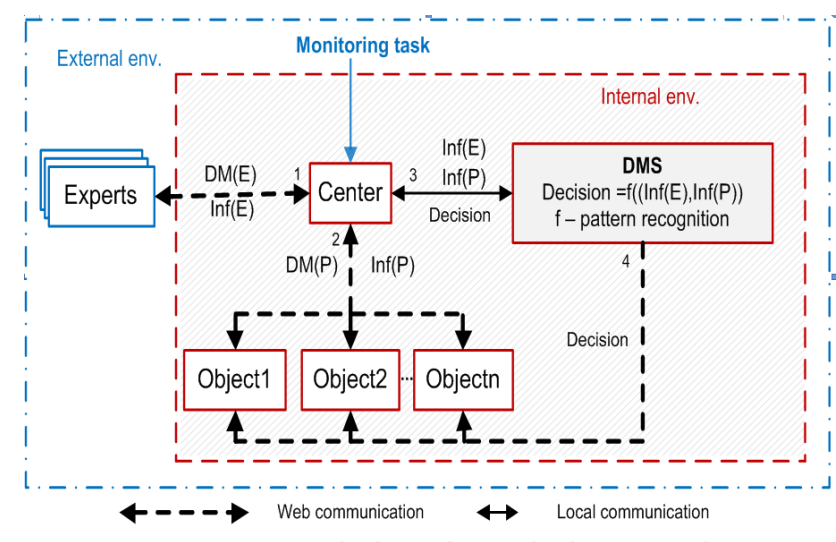

Figure 6. General scheme for monitoring aut omation

\section{Conclusions}

We considered problem of constructing an organizational structure for monitoring of NTC. Here are shown the shortcomings of existing organizational models: the rigidity, complexity of construction and high cost.

It's proposed a virtual model of the organization, focused on the implementation of monitoring, regard less of the size and remoteness of the objects of observation from the center. The algorithm of its construction, based on the representation of the model in the form of attributed tree, is described.

Use of the abbreviated notation of a graph and import of attributes allowed to automate the mapping of any organizational structure in the virtual model, the attribution provided a flexible description of the object, which is relevant to the semantic of the tasks.

The resulting virtual model can be considered as a basis for solving a number of applications for monitoring, including decision-making, knowledge acquisition, visualization of large structures, the construction of dynamic digital maps, integration with other systems, etc.

\section{REFERENCES}

[1] H.Mooney, M.Williamson Bioinvasions and Globalization: Ecology, Economics, Management, and Policy. Oxford University Press, 2010. $-288 \mathrm{p}$

[2] E.P.Odum, G.W.Barret Fundamentals of Ecology. - Brooks Cole, 2004. $-624 \mathrm{p}$

[3] V.E. Sokolov Regional ecological monitoring - M.: Science, 1983. $-263 \mathrm{p}$ 
[4] J.Gross, J.Yellen Graph Theory and Its Applications Shapmann@Hall, 2007.-800 p

[5] M.N.Kirsanov Graphs in MAPLE - M.: FIZMATLIT, 2007. $-168 \mathrm{p}$

[6] V.Krasnoproshin, G.Shakah, A.Valvachev Technology of building decision support systems based on distributed heterogeneous knowledge. Informatics 3, 2004. pp.49-57

[7] V.Krasnoproshin Knowledge-Based Systems: Problems and Solutions . Proceedings of the International Conference on Modeling and Simulation (MS'2006). - Konya: Turkey, 2006.
- Vol. 1. - P. 1-4

[8] V.Krasnoproshin, E.Maksimovich, A.Valvachev, Operational Management of Distributed Socio-Economic System . Proceedings of 10-th International Conference of Pattern Recognition (PRIP'2009), Minsk, Belarus, May 19-21, 2009. -pp. 290-293

[9] G.Shakah, V.V.Krasnoproshin, A.N.Valvachev Fuzzy Approach to Active System Management. . The Fifth International Conference on Neural Networks and Artificial Intelligence. May 27-30, 2008, Minsk Belarus. - P.268-270 